\title{
大鼠眶额叶皮质受损破坏新异性探索行为
}

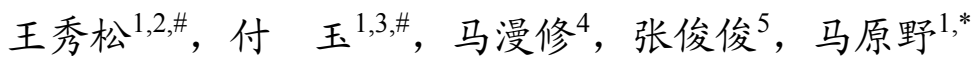 \\ (1. 中国科学院昆明动物研究所 灵长类认知实验室, 云南 昆明 650223 ; 2. 中国科学院研究生院, 北京 100049 ; \\ 3. 云南大学 信息学院电子工程系, 云南 昆明 650091; 4. 中国科学院生物物理研究所 脑与认知科学国家重点实验室, 北京 100101 ; \\ 5. 厦门大学 人工智能研究所, 艺术认知与计算机实验室, 福建 厦门 361005) \\ 摘要: 利用旷场测试和Y-迷宫测试两种行为模型检测了双侧眭额叶（orbitofrontal cortex, OFC）电损伤或假损 \\ 伤雄性 $\mathrm{SD}$ 大鼠的新异性探索行为, 探讨了 $\mathrm{OFC}$ 在大鼠探索新异环境中的作用。旷场测试的结果发现, OFC损伤大 \\ 鼠的行走距离和直立次数较假损组有明显降低; 同时, 在Y-迷宫测试中与假损伤组大鼠相比, OFC损伤大鼠在新 \\ 异臂的访问时间和穿梭次数明显降低。提示眭额叶皮质在大鼠新异性探索行为中起着重要作用。
}

关键词: 眶额叶皮质; 电损伤; 新异性探索; 旷场; Y-迷宫; 大鼠

中图分类号：Q42 文献标识码：A 文章编号：0254-5853-(2009)05-0527-07

\section{Lesions to the Orbitofrontal Cortex Produce the Novelty- Seeking Behavior Deficits in Rats}

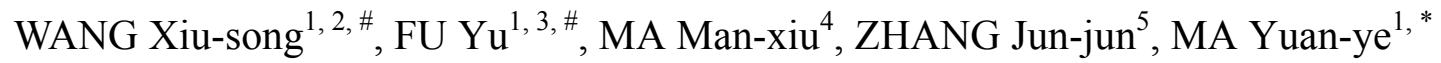 \\ (1. Laboratory of Primate Neuroscience Research, Kunming Institute of Zoology, the Chinese Academy of Sciences, Kunming, 650223, China; \\ 2. Graduate University of the Chinese Academy of Sciences, Beijing, 100049, China; 3. Department of Electronic Engineering, Information School, \\ Yunnan University, Kunming 650091, China; 4. State Key Laboratory of Brain and Cognitive Science, Institute of Biophysics, \\ the Chinese Academy of Sciences, Beijing, 100101, China; 5. Mind, Art and Computation Laboratory, Institute of Artificial \\ Intelligence, School of Information Science and Technology, Xiamen University, Xiamen, 361005, China)
}

\begin{abstract}
We examined the role of the orbitofrontal cortex (OFC) on exploration of the novel environment using the open-field and Y-maze behavioral paradigms to assess the novelty-seeking behavior of the male Sprague-Dawley rats after receiving bilateral electrolytic lesions of the OFC or sham lesions . In the open-field task, the rats with OFC lesions exhibited reduced average ambulation distance and average rearing number when compared with the animals with sham lesions. Moreover, rats with OFC lesions showed less duration of visits and number of entries in the novel arm in the Y-maze task than the control animals. The current findings suggest that the OFC plays an important role on the novelty-seeking behavior in rats.
\end{abstract}

Key words: Orbitofrontal cortex; Electrolytic lesions; Novelty-seeking; Open-field; Y-maze; Rat

新异性探索行为 (novelty-seeking) 是动物的一 种本能, 即动物天生表现出更趋近新异刺激或者对 新异刺激表现出更兴奋的行为特点(Dellu et al, 1996; Renner \& Seltzer, 1991)。这种行为有利于动物 的生存, 比如获取食物、寻找配偶以及释放压力等 (Bardo et al, 1996)。对于新异刺激的定义有多种: 在行为学上, 认为新异刺激是原有刺激的改变; 在
认知水平上, 认为新异刺激是基于先前经验对某个 事件的预测与当前现实之间产生的偏差 (Berns et al, 1997; Bevins et al, 1997)。不管对新异刺激如何定 义, 已经有许多方法被用来检测新异性探索行为 (Dellu et al, 1996; Pawlak et al, 2008), 如旷场行为 (Dellu et al, 1994; Koros et al, 1998)、Y-迷宫探索 (Dellu et al, 1996)、新异物体探索(Besheer \& Bevins,

收稿日期：2009-02-19; 接受日期：2009-08-18

基金项目：国家自然科学基金项目（30470553 和 30770700); CASC 项目 (KSCX1-YW-R-33, YZ200737)

*通讯作者 (Corresponding author), E-mail: yuanma0716@vip.sina.com

\#并列第一作者 (Co-first author), E-mail: wangxs02@post.kiz.ac.cn; fyfuyu@gmail.com 
2000; Ennaceur \& Delacour, 1988; Robinet et al, 1998)、新异气味探索以及新异位置偏好(Nowak et al, 2000)。这些行为模型可以分为两种: 不可逃避 型（如旷场）和可逃避型（如Y-迷宫）。在旷场行 为模型中动物被放入一个新异环境中, 动物无选择 地探索此环境; 而Y-迷宫等行为模型允许动物在熟 悉和新异环境中自由选择。Nowak et al (2000)分别 用了简单旷场、气味丰富旷场以及新异气味识别任 务检测了酒精偏好大鼠和正常大鼠新异性探索行 为, 结果发现酒精偏好大鼠有更高的新异性探索活 动。Tan \& Sui (2003)发现慢性吗啡处理降低了大鼠 对新异物体的探索时间。Dellu et al (1996)用Y-迷 宫研究了大鼠新异性探索行为; 而且他们用这种模 型比较了不同种系小鼠新异性探索行为的差别 (Dellu et al, 2000)。但是, 关于新异性探索行为内在 机制的研究资料并不多。

在神经解剖结构上, 眀额叶皮质 (orbitofrontal cortex, OFC）与前额叶其他部分、内侧颗叶、边缘 系统、杏仁核、感觉以及前运动区有着广泛的神经 纤维联系 (Cavada et al, 2000; Groenewegen \& Uylings, 2000; Hof et al, 1995; Ongur \& Price, 2000; Pandya \& Yeterian, 1990; Schoenbaum et al, 2003; Uylings et al, 2003)。这些广泛的皮层间以及皮层下 核团纤维连接使得OFC能够参与各种感觉、情绪、 行为反应及记忆和决策等高级认知活动 (Petrides, 2007; Rolls, 2004; Schoenbaum \& Roesch, 2005; Schoenbaum \& Setlow, 2001)。在大鼠和狝猴上的行 为学实验结果已经表明 $\mathrm{OFC}$ 参与了行为策略的选 择和执行 (Baxter et al, 2000; Gallagher et al, 1999; Pickens et al, 2003; Pickens et al, 2005; Schoenbaum et al, 1999)。此外, 电生理的研究结果表明狝猴的 OFC区神经元对新异刺激的反应选择性的增强 (Rolls et al, 2005)。脑功能成像实验研究也发现, 当 被试看到新异面孔时, 其大脑OFC区血流量明显增 加, 而且伴随着面孔识别记忆能力的提高(Frey \& Petrides, 2003)。

基于前人的研究工作, 本研究通过电损伤大鼠 双侧OFC, 采用不可逃避的旷场行为模型和可逃避 的Y-迷宫模型, 探讨大鼠OFC受损对其在新异环境 中探索行为的影响, 以初步了解新异性探索行为的 脑机制。

\section{1 材料和方法}

\section{1 实验动物}

健康成年雄性 Sprague-Dawley大鼠, 体重 250 300 g, 购于云南省昆明医学院实验动物中心, 许可证SCXK（滇2005-0008）。所有实验用鼠自购 买回来后, 在本实验室至少适应一周。动物饲养在 $12 \mathrm{~h}$ 明：暗交替(光照时间：07：00-19：00)饲养 间中, 室内温度为 $(23 \pm 1){ }^{\circ} \mathrm{C}$, 大鼠可以自由取食 和饮水, 操作符合动物实验管理条例。

\section{2 电损伤手术}

实验动物被随机分为 $\mathrm{OFC}$ 损伤 (OFC lesion, $n=9$ ）和假损伤（sham lesion, $n=7$ ）两组。参考 Brenhouse et al (2006)的电损伤方法, 但略加修正。 注射成巴比妥钠盐溶液（i.p., $40 \mathrm{mg} / \mathrm{kg}, 40 \mathrm{mg} / \mathrm{mL}$, 上海化工厂）对动物进行麻醉，然后将其固定于立 体定位仪上。沿颅骨中线切开头皮, 并将肌肉等组 织向两侧剥离, 暴露出顽骨, 于左右两侧 $\mathrm{OFC}$ 上 方的领骨各钻一个直径约 $0.5 \mathrm{~mm}$ 的小孔, 立体定 位坐标 (以前囟为坐标原点) 为: 前囟前 $3.7 \mathrm{~mm}$, 中缝左/右旁 $2.4 \mathrm{~mm}$ 。采用直径 $0.3 \mathrm{~mm}$ 单极不锈钢 电极, 套于内径为 $0.32 \mathrm{~mm}$ 石英管内, 仅尖端裸露 出 $0.5 \mathrm{~mm}$ 。将电极垂直于㸚骨下前囟零水平面, 缓 慢插入单侧小孔至预定深度 $4.6 \mathrm{~mm}$ 。本实验中 $\mathrm{OFC}$ 定位坐标参考 Paxinos 和 Watson 大鼠脑立体定位图 谱（Paxinos \& Watson, 1998) 以及我们以前的研究 (Fu et al, 2008; Sun et al, 2006)。接通直流电, 电流 强度 $1 \mathrm{~mA}$, 持续时间 $15 \mathrm{~s}$, 停止通电, 电极停留 15 $\mathrm{s}$ 后取出, 允许离子自由扩散完成。重复同样的操 作损伤对侧 $\mathrm{OFC}$ 。对于假损伤的大鼠，手术操作与 上述一样，不同的是电极深度为 $2 \mathrm{~mm}$ (相对乑骨下 前囟零水平面), 且无电流通过。手术后待动物恢 复 $10 \mathrm{~d}$ 后进行行为学实验。

\section{3 行为学实验}

采用双盲法进行以下 3 种行为学实验。

1.3.1 神经运动功能检测 为了排除 $\mathrm{OFC}$ 损伤对 大鼠前肢或整个身体肌肉强度和运动平衡能力的 影响, 首先对两组大鼠进行神经运动功能检测。检 测在手术后第 $11 \mathrm{~d}$ 进行。方法参考文献(Jeljeli et al, 1999; Ogura et al, 2005), 但略加修正。（1）爬杆实 验: 将大鼠置于距地 $40 \mathrm{~cm}$ 的水平悬空塑料杆 ( 150 $\mathrm{cm} \times 2.7 \mathrm{~cm} \times 2.5 \mathrm{~cm}$, 长 $\times$ 宽 $\times$ 高）中央位点, 记录其 在杆上移动的格数 $(1 \mathrm{~cm} /$ 格 $)$ 和总停留时间。总记 录时间为 $30 \mathrm{~s}$, 若 $30 \mathrm{~s}$ 内大鼠未跌落, 则以 $30 \mathrm{~s}$ 计。

（2）爬网实验：长 $40 \mathrm{~cm} 、$ 宽 $30 \mathrm{~cm}$ 、每个小格长 
宽各 $2 \mathrm{~cm}$ 的网格板, 与地面倾角为 $60^{\circ}$ 且高于地面 $45 \mathrm{~cm}$, 将大鼠头朝向地面倒置于板上, 记录其转 身后向上爬的格数。（3）悬挂实验：将大鼠置于水 平放置的网格板中央, 然后缓慢翻转网格板 $180^{\circ}$, 使网格板面与地面平行, 记录大鼠在网格板上倒置 持续至跌落的时间。若 $30 \mathrm{~s}$ 内大鼠未跌落, 则以 30 $\mathrm{s}$ 计。

1.3.2 旷场实验 旷场实验在 $60 \mathrm{~cm} \times 60 \mathrm{~cm} \times 50 \mathrm{~cm}$ (长 $\times$ 宽 $\times$ 高) 的白色木质行为箱中进行, 木箱底铺 有灰色平滑塑料垫, 在活动箱上方约 $70 \mathrm{~cm}$ 处安装 摄像头。行为箱置于安静有灯光的实验室内。实验 时, 将大鼠放到行为箱的中心处, 观察其自由活动 $5 \mathrm{~min}$, 记录其直立、理毛次数以及粪粒数目, 同时 对大鼠的行为进行录像。采用软件 (由中科院昆明 动物研究所灵长类认知实验室开发) 对录像离线分 析, 计算出大鼠的行走距离。直立次数和行走距离 被作为新异性探索行为的指标(Pawlak et al, 2008)。 在实验中, 伤组中一只大鼠始终停留在活动箱一个 角落不动, 为了减少组内数据的变异性, 该大鼠的 数据被排除。

\subsubsection{Y-迷宫实验 Y-迷宫由灰色 PVC 板制成,} 共 3 个臂, 各个臂夹角为 $120^{\circ}$, 每个臂的尺寸都为 $77 \mathrm{~cm} \times 11 \mathrm{~cm} \times 31 \mathrm{~cm}$ (长×宽 $\times$ 高)。其上方以白光灯 照明, 并于顶部中心安装摄像头, 对大鼠的行为进 行录像。迷宫 3 个臂内分别贴有几个不同白色图 案, 作为视觉线索。为便于区别, 3 个臂被命名为: 起始臂、新异臂和其他臂。

Y-迷宫实验包括两个阶段。第一阶段（训练）: 新异臂被阻挡, 大鼠从起始臂放入, 允许其在迷宫 两个臂 (起始臂和其它臂) 之间自由探索 $15 \mathrm{~min}$ 。 训练结束后取出大鼠并放回笼内。间隔 $2 \mathrm{~min}$ 之后, 开始第二阶段 (测试): 新异臂被打开。同样将大 鼠放入迷宫起始臂, 允许大鼠在 3 个臂之间自由探 索迷宫 $5 \mathrm{~min}$ 。同时在第二阶段对其活动进行录像。 根据录像, 记录大鼠 $5 \mathrm{~min}$ 内分别在 3 个臂的访问时 间和穿梭次数, 然后计算大鼠在各个臂中的停留时 间或穿梭次数占其在 3 个臂中总停留时间或总穿梭 次数的百分比, 这两个指标被用来衡量动物新异性 探索行为。

在两组动物中 3 个臂被随机安排作为新异臂或 其他臂或起始臂, 但对于同一只动物, 3 个臂的安 排在训练和测试时是不变的。根据Dellu et al (2000) 的报道, 本实验选取 $2 \mathrm{~min}$ 为两个阶段的时间间隔。

\section{4 组织学检查}

全部实验结束后对电极插入位点进行组织学 检查, 用含 $1 \%(1 \mathrm{~g}: 100 \mathrm{~mL})$ 亚铁氰化钾 $\mathrm{K}_{4}\left[\mathrm{Fe}(\mathrm{CN})_{6}\right]$ 的 4\%甲醛溶液对动物进行灌流, 然后将脑取出, 置 于 $4 \%$ 甲醛溶液中后固定一周左右。最后用振动切 片机 (浙江象山精密仪器厂) 对脑作连续切片, 切 片厚度为 $80 \mu \mathrm{m}$ 。在解剖镜下观察OFC区域, 如果 定位不在 $\mathrm{OFC}$ 内, 该动物数据将被剔除。

\section{5 数据分析}

数据分析采用统计学软件包SPSS 10.0进行。对 于神经运动功能实验和旷场实验数据, 采用 Mann-Whitney $U$ 独立非参数检验, 进行组间比较。 对于Y-迷宫实验数据, 两组动物对 3 个臂的穿梭次 数的百分比用双因素重复方差分析 (two-way repeated ANOVA)，3个臂两两比较时采用LSD进行 Post-hoc 分析, 采用单因素方差分析 (one-way ANOVA)比较两组间的大鼠在新异臂的穿梭次数百 分比; 由于两组动物在 3 个臂停留时间数据不符合 正态分布, 因此采用配对 (Wilcoxon signed ranks) 和独立 (Mann-Whitney $U$ ) 非参数检验进行数据分 析。 $P<0.05$ 表示数据在统计学上具有显著性差异, $P<0.01$ 为极显著差异。

\section{2 结 果}

\section{1 组织学检查}

组织学检查发现, 损伤组动物中的损伤部位均 在OFC区域内, 但是损伤位点主要分布在内侧和外 侧腹部区域(图1A)。图1B显示在对照组动物中插入 电极的尖端位于背侧。对照组动物在行为实验中无 电流损伤。

\section{2 神经运动功能}

神经运动功能检测的数据表明, 假损伤和损伤 组大鼠的各项运动指标之间无显著差异 $(P>0.05$, Mann-Whitney $U$ )（表1）。在爬格实验中，损伤组 大鼠的爬杆格数和持续时间平均值具有高于假损 伤组的趋势, 可能是由于损伤组大鼠之间存在较大 的组内变异 (方差较大), 因而两者不具有显著性 差异。

\section{3 旷场行为}

旷场实验数据如表 2 所示, 假损伤组大鼠的直 立次数和活动力与损伤组相比, 存在显著差异 $(P<0.05$, Mann-Whitney $U)$, 然而, 其基本行为 的特征参数 (理毛次数和粪便颗粒) 则无显著差异 
$(P>0.05$, Mann-Whitney $U)$ 。由表 2 可以看出, 于损伤组动物。

假损伤组大鼠直立次数和活动力平均值均明显高
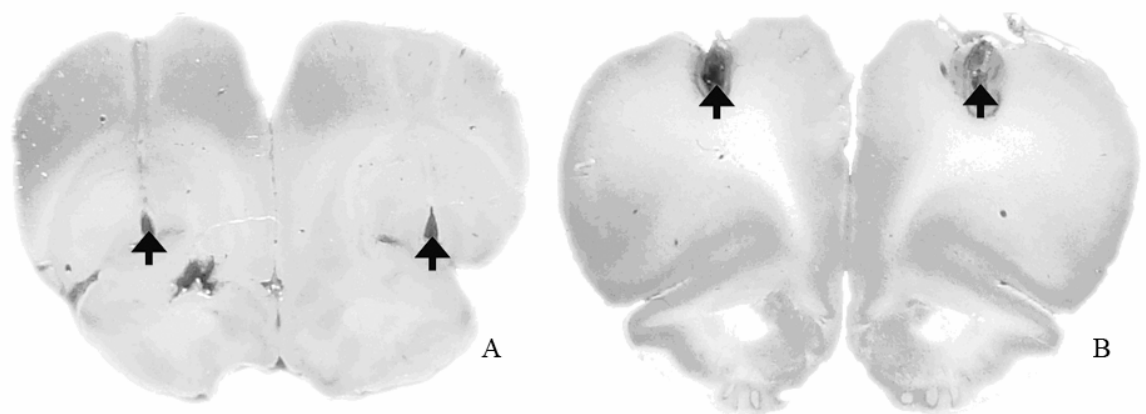

图 1 脑片示插入电极尖端位点

Fig. 1 Photomicrographs of brain sections from the sites of electrode insertion.

$\mathrm{A}$ : 箭头示 $\mathrm{OFC}$ 组动物插入电极尖端的位点; $\mathrm{B}$ : 箭头示对照组动物插入电极尖端的位点。

A: Arrow indicates the sites of the electrode tip in a representative OFC lesion animal; B: Arrow indicates the sites of the electrode tip in a representative Sham lesion animal.

表 1 神经运动能力检测的各项指标

Tab. 1 Summary of the neuromotor parameters measured in the three behavioral tests

\begin{tabular}{|c|c|c|c|}
\hline 神经运动能力 & Neuromotor ability & 假损伤组 Sham group $\quad(n=7)$ & 损伤组 Lesion group $(n=9)$ \\
\hline 爬杆实验 & 格数 Number of grid & $6.8 \pm 1.9$ & $13.6 \pm 4.8$ \\
\hline Wooden beam test & 持续时间 Latency before falling (s) & $4.7 \pm 0.7$ & $11.1 \pm 4.1$ \\
\hline 爬网实验 Hole-board test & 格数 Number of grid & $20.0 \pm 0.0$ & $20.0 \pm 0.0$ \\
\hline 悬挂实验 Suspension test & 持续时间 Latency before falling (s) & $10.4 \pm 3.8$ & $10.0 \pm 2.7$ \\
\hline
\end{tabular}

数据表示为均值 \pm 标准误; 假损伤组与损伤组比较, 无显著差异 ( $P>0.05$, Mann-Whitney $U)$ 。

Each value is expressed as mean $\pm \mathrm{SEM}$. There was no difference between Sham group and Lesion group ( $P>0.05$, Mann-Whitney $U)$.

表 2 旷场实验的各项活动指标

Tab. 2 Summary of the parameters measured in the open-field test

\begin{tabular}{|c|c|c|c|}
\hline 旷场行为 Open-field & 假损伤组 Sham group & $(n=7)$ & 损伤组 Lesion group $(n=8)$ \\
\hline 直立次数 Rearing & $22.3 \pm 3.2^{*}$ & & $10.6 \pm 3.9$ \\
\hline 理毛次数 Grooming & $7.0 \pm 0.8$ & & $6.0 \pm 1$ \\
\hline 粪粒数目 Fecal droppings & $1.7 \pm 0.6$ & & $1.0 \pm 0.8$ \\
\hline 行走距离 Ambulation (m) & $19.4 \pm 1.4^{*}$ & & $12.2 \pm 2.2$ \\
\hline
\end{tabular}

数据表示为均值土标准误; ${ }^{*}$ 示假损伤组与损伤组比较, 差异显著 $(P<0.05$, Mann-Whitney $U)$ 。

Each value is expressed as mean \pm SEM. ${ }^{*}$ indicates that there was a significant difference between Sham group and Lesion group $(P<0.05$, Mann-Whitney $U)$.

\subsection{Y-迷宫}

第二阶段大鼠在各个臂内停留时间百分比如 图2A所示。假损伤组大鼠在新异臂中的停留时间明 显多于在其他臂中的停留时间 $(P=0.018$, Wilcoxon signed ranks test), 但是与起始臂相比无差异 ( $P=0.35$, Wilcoxon signed ranks test); 损伤组大鼠 在新异臂的停留时间与起始臂 $(P=0.051$, Wilcoxon signed ranks test $)$ 和其他臂 $(P=0.48$, Wilcoxon signed ranks test）相比均无差异。另外，假损伤组动物对 新异臂的访问时间显著高于损伤组 $(P=0.016$, Mann-Whitney $U$ )，而与起始臂和其他臂的访问时
间无显著组间差异 ( $P=0.61$ 和 $P=0.96$, Mann-Whitney $U$ )。

第二阶段大鼠在各臂内穿梭次数百分比如图 2B所示。臂之间的差异依赖不同的处理（假损伤和 损伤）或组间（假损伤组和损伤组）差异依赖不同 的臂[臂主效应: $F_{(2,28)}=0.92, P=0.41$, 处理主效应: $F_{(2,28)}=0.77, P=0.4$ ), 交互效应 (处理×臂): $F_{(2,28)}=4.2$, $P=0.025$, Two-way repeated ANOVA]。因此, 单独 组内比较假损伤组和损伤组动物在各个臂之间的 穿梭次数百分比, 发现假损伤组动物在新异臂中的 穿梭次数分别高于起始臂和其他臂 $(P=0.01$ 和 
$P=0.028, \mathrm{LSD})$, 而损伤组动物在各个臂的穿梭次 数之间无显著差异 $(P=0.13, \mathrm{LSD})$ 。同时, 发现损 伤组动物对新异臂的穿梭次数显著低于假损伤组 动物 $\left[F_{(1,14)}=9.2, P<0.01\right.$, one-way ANOVA], 而对另
外两臂的穿梭次数无显著组间差异 [起始臂: $F_{(1,14)}=1.3, P=0.28$, 其 他 臂: $F_{(1,14)}=2.7, P=0.12$, one-way ANOVA]。

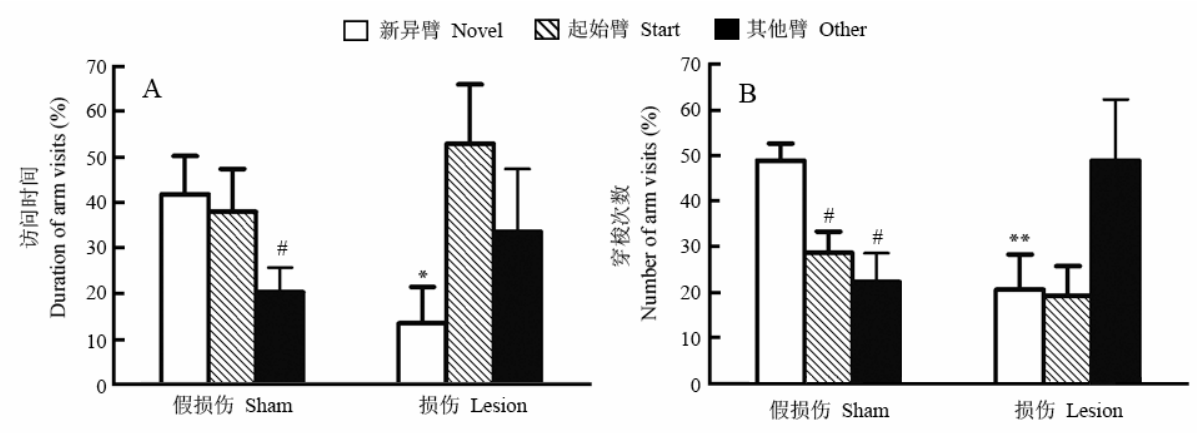

图 2 眀额叶受损破坏大鼠在Y-迷宫中的新异性探索行为

Fig. 2 Orbitofrontal cortex lesions impaired the novelty-seeking behavior of rats in the Y-maze A： $5 \min$ 测试阶段两组大鼠在各个臂中访问时间百分比; B： $5 \min$ 测试阶段两组大鼠在各臂中穿梭次数百分比。

$*$ : 两组动物在新异臂中访问时间比较, 差异显著 $(P<0.05$, Mann-Whitney $U)$; **: 两组动物在新异臂穿梭次数比较, 差异极显著 $(P<0.01$, One-way ANOVA)。\# 表示假损伤组动物在新异臂与熟悉臂中访问时间或穿梭次数比较, 差异显著 $(P<0.05$, Wilcoxon signed ranks test 和LSD)。

\begin{abstract}
A: Percentage of the number of visits made to three arms during the 5-min retention test; B: Percentage of the duration of visits in three arms during the 5-min retention test. *indicates that there was a significant difference in the duration of visits in the novel arm between the two groups $(P<0.05$, Mann-Whitney $U)$; $* *$ indicates that there was an extremely significant difference in the number of entries to the novel arm between the two groups $(P<0.01$, one-way ANOVA); \# indicates that there was a significant difference in the duration of visits or the number of entries between the novel arm and the familiar arms in Sham animals $(P<0.05$, Wilcoxon signed ranks test and LSD).
\end{abstract}

\section{3 讨 论}

Dellu et al (1992)最初用Y-迷宫模型研究了成 年大鼠和老年大鼠的新异性探索行为以及空间辨 别记忆, 此模型是基于大鼠天生具有的探索新异刺 激的倾向而建立的。在两阶段不同间隔时间 (1 min、 $30 \mathrm{~min} 、 2 \mathrm{~h} 、 4 \mathrm{~h} 、 6 \mathrm{~h}$ 和 $24 \mathrm{~h}$ ) 时, 两个年龄组大鼠 行为表现不同, 成年大鼠在间隔 $6 \mathrm{~h}$ 时仍然在新异臂 中有较高的停留时间或穿梭次数, 但是老年动物则 仅仅是在 $1 \mathrm{~min}$ 或 $30 \mathrm{~min}$ 表现出在新异臂有较高的穿 梭次数, 在 3 个臂中的停留时间无差异。而且Dellu et al (1992) 指出停留时间反映动物的“巡查” (inspective) 探索行为, 穿梭次数则反映“获取” (inquisitive) 探索行为, 这是探索行为的两个不同 方面。因此, 在Y-迷宫模型中新异性探索行为的两 个方面受到记忆影响的程度不同。Dellu et al (1996) 又报道在旷场中具有高活动性的大鼠在Y-迷宫新 异臂穿梭次数同样较高, 并认为穿梭次数反映了动 物对环境变化 (比如: 新异臂的出现) 的一种行为 反应。Dellu et al (2000)研究了几个不同品系小鼠 的新异性探索行为, 发现在 $2 \mathrm{~min}$ 的两阶段间隔时,
多数种系小鼠在测试的前 $2 \mathrm{~min}$ 表现出较高的新异 臂探索行为, 但是在较长的间隔 (30 min、1或 $2 \mathrm{~h}$ ), 多数小鼠不是在测试的前 $2 \mathrm{~min}$ 表现出较高的新异 臂探索行为。他们认为间隔时间长可能增加了动物 对新异臂辨别的难度。另外, Nowak et al (2000) 研究发现酒精偏好大鼠在旷场中表现出较高的活 动性; 同样, 在新异性环境条件化位置偏好任务 (和 $\mathrm{Y}$-迷宫类似的自由探索模型) 中对新异箱穿梭次数 也明显高于正常对照大鼠。这些证据都表明Y-迷宫 可以很好地被用来检测动物的新异性探索行为, 但 这种行为受到记忆影响, 因此本实验中选择 $2 \mathrm{~min}$ 作为两阶段时间间隔, 以保证动物对熟悉臂的记忆 程度。Y-迷宫模型允许动物自由选择熟悉臂和新异 臂, 不存在应激对探索行为的影响。另外, 用Y-迷 宫任务研究新异性探索行为得到比旷场模型更多 的信息，比如，可以区分探索行为的两个不同方面。

旷场是不可逃避的新异环境, 已有研究表明不 可逃避的新异环境可能对动物产生应激 (Exner \& Clark, 1993; Robinet et al, 1998), 这种应激能够增加 动物血液中肾上腺素的含量(Piazza et al, 1991), 应 激对动物情绪能够产生影响, 因而可能干扰动物的 
新异性探索行为(Bienkowski et al, 2001)。同时, 已 有研究表明OFC在情绪处理中具有重要作用(Cahill \& McGaugh, 1998; Ongur \& Price, 2000); 而另一些 研究发现OFC损伤并不影响大鼠的厌恶性联合学 习记忆 (Gallagher et al, 1999; Schoenbaum \& Roesch, 2005 ), 从这些研究来看, OFC情绪处理功能可能 不会影响旷场实验结果, 但是需要进一步的研究。

旷场作为新异刺激, 动物可能把饲养环境和旷 场进行比较, 而在Y-迷宫模型中动物必须先熟悉其 中两个臂, 在测试阶段动物把熟悉臂和新异臂进行 比较。这些行为都基于以前的经验与现在环境的比 较才能完成, 因此, 本实验中两种模型所得到的结 果内在机制有相同点。

本研究神经运动功能的数据表明, $\mathrm{OFC}$ 损伤大 鼠与假损伤大鼠相比, 并没有出现明显的神经运动 功能障碍, 而旷场行为研究发现, OFC 损伤的大鼠 在行走距离和直立次数方面较假损伤动物明显降 低, 这与前人的一些研究结果不一致。de Bruin et al (1983)发现损伤Webzob大鼠的 OFC 提高其活动 性; 但Fuchs et al (2004)发现OFC损伤未影响大鼠 的活动性。我们推测, 术后恢复时间、动物种系、 OFC损伤区域以及旷场记录时间等因素都可能导 致以上结果出现差异。Y-迷宫测试结果发现, OFC 损伤大鼠对Y-迷宫新异臂的探索时间和穿梭次数 明显较假损伤动物低。虽然损伤组大鼠在起始臂停 留时间比在新异臂的长以及在其他臂穿梭次数比 在起始臂或新异臂的高, 但因动物在各个臂停留时 间或穿梭次数存在较大的个体变异, 统计结果表明 未到达差异显著性; 假损伤组大鼠在新异臂停留时

\section{参考文献:}

Bardo MT, Donohew RL, Harrington NG. 1996. Psychobiology of novelty seeking and drug seeking behavior [J]. Behav Brain Res, 77(1-2): 23-43.

Baxter MG, Parker A, Lindner CC, Izquierdo AD, Murray EA. 2000. Control of response selection by reinforcer value requires interaction of amygdala and orbital prefrontal cortex [J]. J Neurosci, 20(11): 4311-4319.

Berns GS, Cohen JD, Mintun MA. 1997. Brain regions responsive to novelty in the absence of awareness [J]. Science, 276(5316): 1272-1275.

Besheer J, Bevins RA. 2000. The role of environmental familiarization in novel-object preference [J]. Behav Process, 50(1): 19-29.

Bevins RA, Klebaur JE, Bardo MT. 1997. Individual differences in response to novelty, amphetamine-induced activity and drug discrimination in rats [J]. Behav Pharmacol, 8(2-3): 113-123.

Bienkowski P, Koros E, Kostowski W. 2001. Novelty-seeking behaviour and
间显著多于其他臂, 穿梭次数显著高于起始臂和其 他臂。如上所述, 两模型的结果均表明, OFC损伤 破坏了大鼠的新异性探索行为。前人的研究表明 $\mathrm{OFC}$ 损伤破坏了狝猴对新异物体和熟悉物体的辨 别记忆 (Meunier et al, 1997); 狝猴OFC区一些神经 元能编码新异刺激, 这种记忆能够持续 $24 \mathrm{~h}$, 暗示 这些神经元有助于动物对新异刺激的记忆 (Petrides, 2007; Rolls et al, 2005)。本实验选取 2 min作为两阶 段时间间隔，仍不能排除OFC损伤影响大鼠在第一 阶段对两个臂的长时程编码或记忆, 从而导致在测 试阶段动物不能很好的区分“新异臂”和“熟悉臂”。

Pickens et al（2003，2005）发现不论是在经典条件 化学习建立之后还是在食物贬值实验之后损伤大 鼠OFC, 都能得到同样的结果。这表明OFC损伤的 动物能够进行联合性学习记忆, 但是不能根据条件 变化 (如食物贬值) 采取相应的行为策略 (Gallagher et al, 1999)。由于OFC在行为策略的选择和执行中 具有重要作用(Murray et al, 2007; Schoenbaum \& Setlow, 2001), 而且OFC这种功能和前言中所述的 新异刺激的定义相一致 (Berns et al, 1997; Bevins et al, 1997), Y-迷宫实验结果也可能由于OFC损伤导 致动物不能做出正确的行为决策。

$\mathrm{OFC}$ 与其他皮层区以及皮层下核团有密切的 纤维联系, 决定了它是汇聚、整合及感知所有感觉 刺激和具有多功能性的脑区, 本研究表明OFC的完 整性对于大鼠新异性探索行为非常重要, 旷场与Y迷宫实验结果为此提供了新的证据，但其内在机制 有待研究。

operant oral ethanol self-administration in Wistar rats $[\mathrm{J}]$. Alcohol Alcohol, 36(6): 525-528.

Brenhouse HC, Montalto S, Stellar JR. 2006. Electrolytic lesions of a discrete area within the nucleus accumbens shell attenuate the long-term expression, but not early phase, of sensitization to cocaine [J]. Behav Brain Res, 170(2): 219-223.

Cahill L, McGaugh JL. 1998. Mechanisms of emotional arousal and lasting declarative memory [J]. Trends Neurosci, 21(7): 294-299.

Cavada C, Company T, Tejedor J, Cruz-Rizzolo RJ, Reinoso-Suarez F. 2000. The anatomical connections of the macaque monkey orbitofrontal cortex [J]. Cereb Cortex, 10(3): 220-242.

de Bruin JPC, Vanoyen HGM, Vandepoll N. 1983. Behavioral-changes following lesions of the orbital prefrontal cortex in male-rats [J]. Behav Brain Res, 10(2-3): 209-232.

Dellu F, Contarino A, Simon H, Koob GF, Gold LH. 2000. Genetic differences in response to novelty and spatial memory using a two-trial 
recognition task in mice [J]. Neurobiol Learn Mem, 73(1): 31-48.

Dellu F, Mayo W, Cherkaoui J, Lemoal M, Simon H. 1992. A 2-trial memory task with automated recording: Study in young and aged rats [J]. Brain Res, 588(1): 132-139.

Dellu F, Mayo W, Vallee M, Lemoal M, Simon H. 1994. Reactivity to novelty during youth as a predictive factor of cognitive impairment in the elderly: A longitudinal-study in rats [J]. Brain Res, 653(1-2): 51-56.

Dellu F, Piazza PV, Mayo W, Le MM, Simon H. 1996. Novelty-seeking in rats: Biobehavioral characteristics and possible relationship with the sensation-seeking trait in man [J]. Neuropsychobiology, 34(3): 136-145.

Ennaceur A, Delacour J. 1988. A new one-trial test for neurobiological studies of memory in rats. 1: Behavioral data [J]. Behav Brain Res, 31(1): 47-59.

Exner M, Clark D. 1993. Behavior in the Novel environment predicts responsiveness to D-amphetamine in the rat: A multivariate approach [J]. Behav Pharmacol, 4(1): 47-56.

Frey S, Petrides M. 2003. Greater orbitofrontal activity predicts better memory for faces [J]. Eur J Neurosci, 17(17): 2755-2758.

Fu Y, Chen YM, Zeng T, Peng YP, Tian SH, Ma YY. 2008. Delta EEG activity in left orbitofrontal cortex in rats related to food reward and craving [J]. Zool Res, 29(3): 260-264. [付 玉, 陈艳梅, 曾 涛, 彭 沿平, 田绍华, 马原野. 2008. 食物奖赏和渴求行为相关的大鼠左 侧眭额叶皮质 Delta 频段脑电活动. 动物学研究, 29(3): 260-264.]

Fuchs RA, Evans KA, Parker MP, See RE. 2004. Differential involvement of orbitofrontal cortex subregions in conditioned cue-induced and cocaine-primed reinstatement of cocaine seeking in rats [J]. J Neurosci, 24(29): 6600-6610.

Gallagher M, McMahan RW, Schoenbaum G. 1999. Orbitofrontal cortex and representation of incentive value in associative learning [J]. J Neurosci, 19(15): 6610-6614.

Groenewegen HJ, Uylings HB. 2000. The prefrontal cortex and the integration of sensory, limbic and autonomic information [J]. Prog Brain Res, 126: 3-28.

Hof PR, Mufson EJ, Morrison JH. 1995. Human orbitofrontal cortex: Cytoarchitecture and quantitative immunohistochemical parcellation [J]. J Comp Neurol, 359(1): 48-68.

Jeljeli M, Strazielle C, Caston J, Lalonde R. 1999. Effects of electrolytic lesions of the lateral pallidum on motor coordination, spatial learning, and regional brain variations of cytochrome oxidase activity in rats [J]. Behav Brain Res, 102(1-2): 61-71.

Koros E, Piasecki J, Kostowski W, Bienkowski P. 1998. Saccharin drinking rather than open field behaviour predicts initial ethanol acceptance in Wistar rats [J]. Alcohol Alcohol, 33(2): 131-140.

Meunier M, Bachevalier J, Mishkin M. 1997. Effects of orbital frontal and anterior cingulate lesions on object and spatial memory in rhesus monkeys [J]. Neuropsychologia, 35(7): 999-1015.

Murray EA, O'Doherty JP, Schoenbaum G. 2007. What we know and do not know about the functions of the orbitofrontal cortex after 20 years of cross-species studies [J]. J Neurosci, 27(31): 8166-8169.

Nowak KL, Ingraham CM, Mckinzie DL, Mcbride WJ, Lumeng L, Li TK, Murphy JM. 2000. An assessment of novelty-seeking behavior in alcohol-preferring and nonpreferring rats [J]. Pharmacol Biochem Behav, 66(1): 113-121.

Ogura T, Ogata M, Akita H, Jitsuki S, Akiba L, Noda K, Hoka S, Saji M. 2005. Impaired acquisition of skilled behavior in rotarod task by moderate depletion of striatal dopamine in a pre-symptomatic stage model of Parkinson's disease [J]. Neurosci Res, 51(3): 299-308.
Ongur D, Price JL. 2000. The organization of networks within the orbital and medial prefrontal cortex of rats, monkeys and humans [J]. Cereb Cortex, 10(3): 206-219.

Pandya DN, Yeterian EH. 1990. Prefrontal cortex in relation to other cortical areas in rhesus monkey: architecture and connections [J]. Prog Brain Res, 85: 63-94.

Pawlak CR, Ho YJ, Schwarting RK. 2008. Animal models of human psychopathology based on individual differences in novelty-seeking and anxiety [J]. Neurosci Biobehav Rev, 32(8): 1544-1568.

Paxinos G, Watson C. 1998. The Rat Brain in Stereotaxic Coordinates.. [M]. 4th ed. San Diego: Academic Press.

Petrides M. 2007. The orbitofrontal cortex: novelty, deviation from expectation, and memory [J]. Ann N Y Acad Sci, 1121: 33-53.

Piazza PV, Maccari S, Deminiere JM, Le MM, Mormede P, Simon H. 1991. Corticosterone levels determine individual vulnerability to amphetamine self-administration [J]. Proc Natl Acad Sci USA, 88(6): 2088-2092.

Pickens CL, Saddoris MP, Gallagher M, Holland PC. 2005. Orbitofrontal lesions impair use of cue-outcome associations in a devaluation task [J]. Behav Neurosci, 119(1): 317-322.

Pickens CL, Saddoris MP, Setlow B, Gallagher M, Holland PC, Schoenbaum G. 2003. Different roles for orbitofrontal cortex and basolateral amygdala in a reinforcer devaluation task [J]. J Neurosci, 23(35): 11078-11084.

Renner MJ, Seltzer CP. 1991. Molar characteristics of exploratory and investigatory behavior in the rat (Rattus norvegicus) [J]. J Comp Psychol, 105(4): 326-339.

Robinet PM, Rowlett JK, Bardo MT. 1998. Individual differences in novelty-induced activity and the rewarding effects of novelty and amphetamine in rats [J]. Behav Process, 44(1): 1-9.

Rolls ET. 2004. The functions of the orbitofrontal cortex [J]. Brain Cogn, 55(1): 11-29.

Rolls ET, Browning AS, Inoue K, Hernadi I. 2005. Novel visual stimuli activate a population of neurons in the primate orbitofrontal cortex $[\mathrm{J}]$. Neurobiol Learn Mem, 84(2): 111-123.

Schoenbaum G, Chiba AA, Gallagher M. 1999. Neural encoding in orbitofrontal cortex and basolateral amygdala during olfactory discrimination learning [J]. J Neurosci, 19(5): 1876-1884.

Schoenbaum G, Roesch M. 2005. Orbitofrontal cortex, associative learning, and expectancies [J]. Neuron, 47(5): 633-636.

Schoenbaum G, Setlow B. 2001. Integrating orbitofrontal cortex into prefrontal theory: Common processing themes across species and subdivisions [J]. Learn Mem, 8(3): 134-147.

Schoenbaum G, Setlow B, Ramus SJ. 2003. A systems approach to orbitofrontal cortex function: recordings in rat orbitofrontal cortex reveal interactions with different learning systems [J]. Behav Brain Res, 146(1-2): 19-29.

Sun NL, Li YX, Tian SW, Lei YL, Zheng JW, Yang JZ, Sui N, Xu L, Pei G, Wilson FAW, Ma YY, Lei H, Hu XT. 2006. Dynamic changes in orbitofrontal neuronal activity in rats during opiate administration and withdrawal [J]. Neuroscience, 138(1): 77-82.

Tan BP, Sui N. 2003. The effect of chronic morphine treatment on the novelty seeking behavior of rats [J]. Chn J Drug Depend, 12(4): 255-257. [谭北平, 隋 南. 2003. 吗啡慢性给药对大鼠新颖寻求行 为的促进作用. 中国药物依赖性杂志, 12(4): 255-257.]

Uylings HBM, Groenewegen HJ, Kolb B. 2003. Do rats have a prefrontal cortex [J]? Behav Brain Res, 146(1-2): 3-17. 